University of Nebraska - Lincoln

DigitalCommons@University of Nebraska - Lincoln

$1-2018$

\title{
Temporal Variation in Predation Risk May Explain Daily Rhythms of Foraging Behavior in an Orb-Weaving Spider
}

\author{
J. Colton Watts \\ University of Nebraska-Lincoln, wattsj@huskers.unl.edu \\ Thomas C. Jones \\ East Tennessee State University \\ Ashley Herrig \\ East Tennessee State University \\ Madeleine Miller \\ East Tennessee State University \\ Brigitte Tenhumberg \\ University of Nebraska - Lincoln, btenhumberg2@unl.edu
}

Follow this and additional works at: https://digitalcommons.unl.edu/bioscifacpub

Part of the Biology Commons

Watts, J. Colton; Jones, Thomas C.; Herrig, Ashley; Miller, Madeleine; and Tenhumberg, Brigitte, "Temporal Variation in Predation Risk May Explain Daily Rhythms of Foraging Behavior in an Orb-Weaving Spider" (2018). Faculty Publications in the Biological Sciences. 805.

https://digitalcommons.unl.edu/bioscifacpub/805

This Article is brought to you for free and open access by the Papers in the Biological Sciences at DigitalCommons@University of Nebraska - Lincoln. It has been accepted for inclusion in Faculty Publications in the Biological Sciences by an authorized administrator of DigitalCommons@University of Nebraska - Lincoln. 


\title{
Temporal Variation in Predation Risk May Explain Daily Rhythms of Foraging Behavior in an Orb-Weaving Spider
}

\author{
J. Colton Watts, ${ }^{1, *}$ Thomas C. Jones, ${ }^{2}$ Ashley Herrig, ${ }^{2}$ Madeleine Miller, ${ }^{2}$ \\ and Brigitte Tenhumberg ${ }^{1}$
} 1. School of Biological Sciences, University of Nebraska-Lincoln, Lincoln, Nebraska 68588; 2. Department of Biological Sciences,
East Tennessee State University, Johnson City, Tennessee 37614

Submitted December 15, 2016; Accepted July 21, 2017; Electronically published November 9, 2017

Online enhancements: appendix. Dryad data: http://dx.doi.org/10.5061/dryad.959g2.

\begin{abstract}
Daily rhythms occur in numerous physiological and behavioral processes across an immense diversity of taxa, but there remain few cases in which mechanistic links between rhythms of trait expression and organismal fitness have been established. We construct a dynamic optimization model to determine whether risk allocation provides an adaptive explanation for the daily foraging rhythm observed in many species using the orb-weaving spider Cyclosa turbinata as a case study. Our model predicts that female C. turbinata should generally start foraging at lower levels of energy reserves (i.e., should be less bold) during midday when predators are most abundant. We also find that individuals' foraging efficacy determines whether daily rates of encounters with predators or prey more strongly influences boldness under high risk. The qualitative model predictions are robust to variation in our parameter estimates and likely apply to a wide range of taxa. The predictions are also consistent with observed patterns of foraging behavior under both laboratory and field conditions. We discuss the implications of our study for understanding the evolution of daily rhythms and the importance of model predictions for interpreting empirical studies and generating additional hypotheses regarding behavioral evolution.
\end{abstract}

Keywords: daily rhythm, risk allocation, predation, foraging, predatorprey interaction.

\section{Introduction}

Daily rhythms of physiology and behavior are a nearly ubiquitous form of temporal organization in living systems. Not only are daily rhythms extremely taxonomically widespread, but they are also expressed across a variety of physiological and behavioral traits within taxa (DeCoursey 2004). Although a variety of proximate mechanisms may be used to modify trait expression over the daily cycle (e.g., exoge-

* Corresponding author; e-mail: j.colton.watts@gmail.com.

Am. Nat. 2018. Vol. 191, pp. 74-87. (C) 2017 by The University of Chicago. 0003-0147/2018/19101-57447\$15.00. All rights reserved. DOI: $10.1086 / 694775$ nous cues, endogenous rhythms [sensu Aschoff 1981a], and their interactions [Rietveld et al. 1993]), the resulting pattern of changes in trait expression (the overt, or measured rhythm sensu Rietveld et al. 1993) typically corresponds to (or even anticipates) changes in external environmental conditions that occur over the course of the day, such as changes in the availability of resources or the intensity of risks. Consequently, daily rhythms are widely assumed to be adaptive strategies for coping with daily changes in environmental conditions (DeCoursey 2004; Johnson 2005; Vaze and Sharma 2013). Although this assumption has motivated decades of highly productive research on the proximate mechanisms that contribute to overt daily rhythms of trait expression, such as endogenous circadian rhythms, evidence for the adaptive significance of daily rhythms remains surprisingly limited, as rigorous manipulative tests have proven difficult (reviewed in DeCoursey 2004; Johnson 2005; Vaze and Sharma 2013).

Much of the putative evidence for an adaptive value of daily rhythms rests on the assertion that daily changes in trait expression should reflect changes in an environmental factor with a plausible effect on the fitness consequences of the trait. For example, it is well known that honeybees time their foraging activities to match daily cycles of nectar and pollen availability (reviewed in Moore 2001). Similarly, male Texas field crickets sing louder, longer, and more frequently during periods of the day when receptive females are abundant relative to parasitoid flies (Bertram et al. 2004). However, rhythmic changes in trait expression may be correlated with changing environmental conditions through mechanisms unrelated to changing fitness consequences of trait expression per se (Johnson 2005). This may be the case if the daily rhythm derives from intrinsic (e.g., organizational) constraints or if altering the trait rhythm also produces correlated, nonrhythmic shifts in traits associated with fitness (Beaver et al. 2003). Thus, more rigorous tests of the adap- 
tive value of a daily rhythm typically measure the fitness consequences of experimentally decoupling patterns of trait expression and environmental change (but see Vaze and Kumar 2013 for a discussion of empirical approaches). In practice, this requires demonstrating that (i) changes in the pattern of trait expression affect fitness and (ii) the fitness effects of changes in the pattern of trait expression depend on the pattern of environmental change. While the first criterion assesses the potential for natural selection to favor the observed rhythm, the second criterion determines whether daily patterns of environmental change per se provide the mechanism for selection on the rhythm (Vaze and Sharma 2013).

Very few studies demonstrate environmentally determined fitness advantages of daily rhythms by meeting both of these criteria. For example, in cyanobacteria, the outcome of competition between strains that differ in the period of their endogenous rhythm (and therefore presumably their overt rhythm; Pittendrigh and Daan 1976; Aschoff 1981b; Emens et al. 2009) depends on the correspondence between each strain's rhythm and the length of the light: dark cycle (Ouyang et al. 1998). Similarly, in a Drosophila parasitoid, a daily rhythm of oviposition behavior increases fitness by providing competitively inferior larvae with a developmental advantage when hosts are parasitized again later in the day by a competitively superior congener (Fleury et al. 2000). However, to our knowledge, the work of Fleury et al. remains one of the only studies that directly assesses the fitness consequences of variation in daily temporal niche separation for competitors, and the ecological mechanism through which rhythms confer competitive advantages in cyanobacteria is yet unresolved. Consequently, our understanding of the evolution of daily rhythms is perhaps most limited by the lack of studies that investigate precise yet generalizable ecological mechanisms through which rhythmic changes in trait expression may provide fitness advantages. Addressing this knowledge gap requires explicitly defining the mechanisms through which daily environmental changes may affect trade-offs of trait expression and determining whether these mechanisms are likely to produce selection for rhythms of trait expression under natural conditions. Importantly, this focus on the ecological mechanisms favoring the evolution of daily rhythms does not require assumptions about the specific proximate mechanisms through which daily changes in trait expression are achieved (Enright 1970). Instead, characterizing the optimal pattern of trait expression under natural conditions may provide predictions about the nature of proximate mechanisms that evolve in response to selection for the daily rhythm.

It is widely acknowledged that temporal variation in predation risk can drive patterns of behavior. This mechanism of selection for shifts in behavior requires only that there be trade-offs between predation risk and fitness-increasing activities (e.g., searching for mates or foraging) and that the intensity of predation risk relative to fitness benefits varies over time (Lima and Bednekoff 1999). For example, Allenby's gerbils increase vigilance and leave behind more food under brighter moon phases that increase the riskiness of foraging (Kotler et al. 2010). Similarly, roe deer show greater levels of vigilance during the period of the season when human hunting occurs (Sönnichsen et al. 2013). Given the ubiquity of daily rhythms, it seems likely that predation risk also varies over the daily cycle for many organisms. Thus, selection for daily rhythms may in many cases arise from changes in foragingpredation risk trade-offs that occur over the daily cycle. Despite this, there remain few studies that rigorously assess the plausibility of temporal changes in foraging-predation risk trade-offs for generating daily rhythms of behavior by testing the predictions of mechanistic models under natural conditions (but see Rosland and Giske 1994; Metcalfe et al. 1999; Tarling et al. 2000; Jensen et al. 2006), and to our knowledge no such studies have been conducted for terrestrial organisms.

Here, we develop a state-dependent dynamic optimal foraging model to determine the extent to which adaptive responses to temporal variation in predation risk provide a general explanation for daily rhythms of foraging behavior. We parameterize the model using empirical data from an orb-weaving spider, Cyclosa turbinata, and compare the model predictions to rhythms of behavior observed in the laboratory (Watts et al. 2014) and under natural conditions (this study). We find that the qualitative pattern of foraging predicted by the model represents a robust solution to the foraging-predation risk trade-off and is likely applicable to a wide range of species. Moreover, the model predicts that the effect of individual differences in foraging performance on patterns of foraging decisions depends on prey abundance and predation risk. This arises because the optimal response of the pattern of foraging decisions to changing environmental conditions differs for more and less effective foragers.

\section{The Model}

We used stochastic dynamic programming (SDP; Clark and Mangel 2000) to determine whether variation in the tradeoff between predation risk and food availability produces daily rhythms in foraging activity of a sit-and-wait predator (we briefly consider how the model differs for predators that must actively search for prey in the appendix, available online). SDPs are discrete-time models that can incorporate information about individuals' internal and external state, time constraints, and the ecological and physiological consequences of decisions to predict strategies that maximize fitness. Specifically, SDP models determine for each combination of states the behavior that maximizes expected fitness at the end of a period of interest (e.g., the end of the daily cycle).

We begin by dividing the daily cycle into time steps equal in length to the average time needed to complete a 
single foraging bout (i.e., the handling time, $h$ ). For simplicity, we assume that an individual that does not suffer predation as a result of foraging in a given time step always survives to the subsequent time step (i.e., there are no other sources of mortality). Although we acknowledge that other sources of mortality affect the absolute expected fitness at the end of the day, such fitness decrements are assumed to occur for both resting and foraging spiders and therefore should not affect the decision to forage. Thus, in each time step, we consider the following hierarchy of events. The individual encounters (i.e., has an opportunity to attempt to consume) a prey item with probability $R(t)$. If no prey item is encountered, the individual's energetic reserves are reduced by the metabolic cost of resting for one time step, $c_{1}$. If instead the individual encounters a prey item, the individual must choose whether to forage or rest. This is the decision over which the model optimizes fitness. If the individual chooses to rest, the individual's energetic reserves are reduced by the metabolic cost of resting for one time step, $c_{1}$, as if no prey had been encountered. If the individual forages, then it must survive predation risk associated with foraging (survival probability $=1-\mu(t)$ ) that varies over the daily cycle (i.e., across time steps). If the individual survives this risk, then it captures the prey item with probability $f$. An individual that succeeds in capturing a prey item increases its future energetic state by one unit, minus the metabolic cost of foraging, $c_{2}$ (where $c_{2}>c_{1}$ ). If the individual fails to capture the prey item (probability $1-f$ ), it gains no energy but pays the metabolic cost of foraging $c_{2}$.

It is worthwhile to note that this scenario differs from that in which predators must search for their prey. Searching predators must instead determine the optimal decision, to forage or to rest, at each time of day regardless of whether prey have been encountered and according to both predation risk and the likelihood that prey would be encountered and then successfully captured if the individual opted to search. We briefly outline these differences and their implications for expected patterns of behavior in the appendix.

\section{State Space}

We assume that an individual's foraging behavior depends only on energetic reserves $(i)$ and the time of day $(t)$. For each combination of states, fitness is maximized over the behavioral decision. Time of day is an external state variable that determines (i) the time remaining to forage, (ii) the likelihood that prey are encountered, and (iii) the magnitude of additional predation risk due to foraging.

\section{Fitness Currency}

We assume that individuals modify their foraging behavior to maximize fitness at the end of each day, which ignores possible effects of an organism's age on the daily foraging rhythm. We further assume that fitness is a sigmoidal function of energetic reserves (Clark and Mangel 2000). Energetic reserves at the end of the daily cycle may be associated with fitness for at least two reasons. First, if energetic reserves fall below a critical level, the individual will starve, thereby forfeiting all future reproductive potential. Second, if energetic reserves exceed those needed for survival, relative fitness may be largely determined by the effect of energy reserves on key components of fitness such as fecundity or growth rate. Although the precise shape of the relationship between daily energetic reserves and fitness likely varies among systems, in general, the relationship is likely to be sigmoidal when considering the full range of possible energetic reserves.

\section{Predation Risk and Prey Availability}

We assume that individuals that forage suffer predation risk, $\mu(t)$, due to foraging activities. This mortality risk depends on the probability of encountering a predator during the time step, which is a function of the time of day, $P(t)$, and the probability $m$ that an encounter with a predator results in predation (i.e., $\mu(t)=m \times P(t))$. This parameterization encompasses situations in which predation must be inferred by sampling the temporal distributions of the predators of the focal species. Decomposing $\mu(t)$ into two separate processes is unnecessary if temporal distribution of $\mu(t)$ can instead be estimated directly. For our case study (see Parameterizing the Model), we inferred predation risk and prey intake from the temporal distributions of the predators. Because we did not have strong estimates for $m$, we varied this parameter to examine its effect on the model predictions. Similarly, we assume that prey consumption depends on both the probability of encountering a prey item, $R(t)$, and the prey capture efficacy, $f$ (i.e., the probability that the individual successfully captures the encountered prey item if the individual chooses to attack). Because we also did not have strong estimates for $f$, we varied this parameter to examine its effect on the model predictions.

We also assume that individuals know the values of predation risk $\mu(t)$ and prey availability $R(t)$. The temporal pattern of relative predator and prey abundance within the day is typically associated with reliable environmental cues (e.g., changes in light, temperature, or humidity) and is likely to be fairly consistent across days. Consequently, it is reasonable to assume that selection could favor daily changes in behavior associated with environmental predictors of relative predation risk and prey abundance. However, the overall magnitudes of $\mu(t)$ and $R(t)$ may vary across days as well. Like the temporal pattern within days, changes in magnitude across days may be in part associated with environmental cues or show some degree of consistency (i.e., temporal autocorrela- 
tion). It is also likely that individuals can gather information about the magnitudes of $\mu(t)$ and $R(t)$ over the course of the day by monitoring previous encounter rates. Although we do not explicitly consider situations in which individuals do not know these environmental parameters, even an extreme scenario in which individuals rely entirely on previous encounters to estimate environmental parameters using simple memory rules (e.g., sample and forget; Mangel 1990) would likely result in reasonable estimates of environmental parameters (McNamara and Houston 1985; Mangel 1990) and therefore differ from our results primarily in the rapidity with which behavior changes over the day (as individuals must sample the environment to detect changing conditions; Mangle 1990).

\section{Metabolic Costs}

We assume that a unit of energetic reserves in our model corresponds to the energetic content of a typical prey item. If the value of the energetic content of prey and the basal metabolic rate of the focal species are known, these values can be used to estimate the fraction of an energy unit lost in each time step due to resting metabolism, $c_{1}$. We further assume that foraging incurs additional metabolic costs associated with locomotion and handling of prey. Consequently, we assume that the metabolic cost of foraging, $c_{2}$, is a scalar multiple $s$ of the basal metabolic rate, $c_{1}$, such that $c_{2}>c_{1}$. Individuals choosing to forage must pay the additional metabolic cost independent of whether foraging is successful.

\section{Dynamic Programming Equation}

Assuming that the time step is equal in length to the handling time of a single prey item $h$, independent of foraging decision, and that individuals are in principle capable of foraging for some portion of the day $l$, the model consists of $l / h$ time steps. The model includes the state variables time $(t=1,2,3, \ldots, l / h)$ and energy reserves $(i=0,1,2, \ldots, 100)$, where energy reserves represent the percentage of maximum possible reserves. Expected fitness at the end of the day is therefore a function of current time and energy reserves, $F(i, t)$.

We consider only two actions, foraging and resting. If no prey item is available, the individual has no choice but to rest. If a prey item is available, the individual may choose to forage or ignore the prey item (rest). Each of these actions corresponds to a payoff (payoff forage $_{\text {or payoff }}$ rest). The payoff of foraging depends on whether the individual survives predation $(1-\mu(t))$ and succeeds in capturing the prey item $(f)$.
Thus, the payoffs for foraging (payoff $f_{\text {forage }}$ ) and resting (payoff rest $_{\text {) }}$ ) are as follows:

$$
\begin{aligned}
\text { payoff }_{\text {forage }}= & (1-\mu(t)) \times\left(f \times F\left(i+1-c_{2}, t+1\right)\right. \\
& \left.+(1-f) \times F\left(i-c_{2}, t+1\right)\right), \\
\text { payoff }_{\text {rest }}= & F\left(i-c_{1}, t+1\right),
\end{aligned}
$$

where the value of $F$ for noninteger values of energetic state are estimated using linear interpolation (Clark and Mangel 2000). We represent this scenario in the following dynamic programming equation that maximizes fitness $F$ over the behavioral decision, forage or rest:

$$
\begin{aligned}
F(i, t)= & (1-R(t)) \times \text { payoff }_{\text {rest }}+R(t) \\
& \times \max \left(\text { payoff } \text { forage }_{\text {, }} \text {, payoff }{ }_{\text {rest }}\right) .
\end{aligned}
$$

\section{A Case Study of the Orb-Weaving Spider Cyclosa turbinata}

\section{The Biological Scenario}

Female Cyclosa turbinata construct small ( 30-cm-diameter) orb webs that they decorate with a conspicuous column of detritus and prey remains. Females perform their sit-and-wait foraging from the center of the web (the web hub), where they remain aligned with the web decoration (fig. 1). Studies on

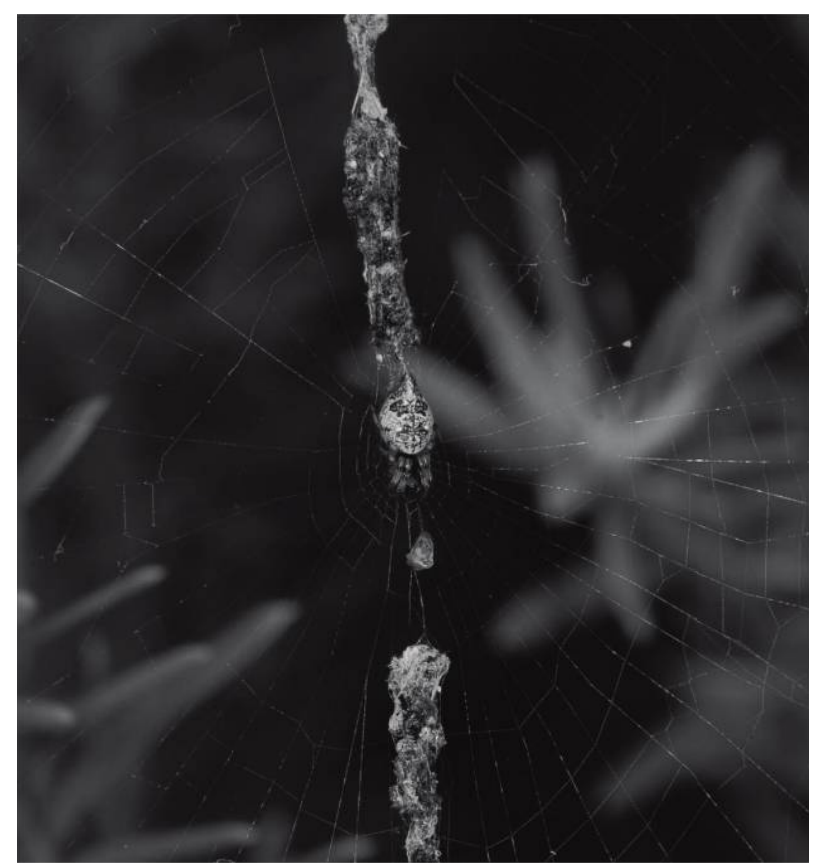

Figure 1: A female Cyclosa turbinata resting at the web hub. The web decoration extending above and below the spider is composed of prey remains and detritus. Photo courtesy of Richard Bradley. Reprinted from Watts et al. (2014) with permission of the publisher. 
other Cyclosa species have shown that the web decoration reduces the ability of hymenopteran predators to locate the spider at the web hub, thereby redirecting attacks away from the spider and toward the decoration (Chou et al. 2005; Gonzaga and Vasconcellos-Neto 2005; Tseng and Tso 2009). Hymenopterans, specifically wasps, are a primary source of predation and parasitism for orb-weaving spiders (Blackledge et al. 2003).

Female C. turbinata remain at the web hub continuously and are thus capable of ensnaring prey at nearly all times of day (Watts et al. 2014). However, ensnared prey can escape the web if not subdued, and females must leave the safety of the decoration to subdue prey. Consequently, increases in energetic reserves (which increase fecundity in C. turbinata; Spiller 1984) likely come at a direct cost of increased exposure to hymenopteran predators, and females must balance these demands to maximize fitness. Because visually oriented hymenopteran predators are expected to be primarily diurnal, female C. turbinata are expected to largely ignore prey during the daytime and instead forage at night, when the movement associated with subduing prey is less likely to reveal the spider's location to predators. Accordingly, previous work shows that C. turbinata maintained in the laboratory more frequently attack simulated prey during the night (Watts et al. 2014). However, whether this daily rhythm of foraging behavior can be driven by a tradeoff between foraging and predation risk alone has yet to be tested directly using data collected under natural conditions. We parameterize our general model using patterns of predator and prey abundance from typical C. turbinata habitat (Moore et al. 2016) to determine whether the optimal foraging policy changes over the course of the day and under what conditions this is expected to be the case. We then compare the qualitative model predictions to data from a previous laboratory study of the daily foraging rhythm in C. turbinata (Watts et al. 2014) as well as new data from observations of foraging behavior of $C$. turbinata under natural conditions (see Foraging Observations).

\section{Parameterizing the Model}

Parameters used in our case study are presented in table 1 along with their definitions, default values, and sources. We calculated the handling time $h$ to be $85.8 \pm 79.5 \mathrm{~s}$ (mean $\pm \mathrm{SD}$ ) across all attacks observed in the field $(n=110)$. We made the simplifying assumption that females can ensnare prey at any time of day and therefore divided the 24-h day into 1,007 time steps, beginning at 0600 hours when females typically finish the daily replacement of the sticky capture spiral of their webs (Moore et al. 2016). This ignores the period of each night females spend replacing the capture spiral, during which time they presumably cannot ensnare prey.

We estimated the probability, as a function of time of day, of encountering potential hymenopteran predators and flying insect prey in the natural habitat of $C$. turbinata (i.e., $R(t)$ and $P(t))$ from encounter rates published in a previous study (Moore et al. 2016). We converted the published estimates of the average encounter rates of potential predators (hymenoptera) and prey per 3-h interval of the day (see detailed methods in Moore et al. 2016) to encounter probabilities per time step of the model using the formula $p$ (event) $=1-e^{(-r t)}$, where $t$ is a single-model time step and $r$ is the average encounter rate per 3-h interval divided

Table 1: Parameters used in the stochastic dynamic programming model of daily foraging behavior in Cyclosa turbinata

\begin{tabular}{|c|c|c|c|}
\hline Parameter & Definition & Value(s) & Source \\
\hline Units of $t$ & $\begin{array}{l}\text { Time required to subdue one prey } \\
\text { item (i.e., handling time) }\end{array}$ & $85.8 \mathrm{~s}$ & This article \\
\hline$R(t)$ & $\begin{array}{l}\text { Probability of encountering } \\
\text { a prey item }\end{array}$ & $\begin{array}{c}\max \left(0,-6.131 \times 10^{-2}+1.207 \times 10^{-3} t-\right. \\
\left.\quad 1.984 \times 10^{-6} t^{2}+8.367 \times 10^{-10} t^{3}\right)\end{array}$ & $\begin{array}{l}\text { Moore et al. 2016; function } \\
\text { derived in this article }\end{array}$ \\
\hline$P(t)$ & $\begin{array}{l}\text { Probability of encountering } \\
\text { a predator }\end{array}$ & $\begin{array}{c}\max \left(0,-4.086 \times 10^{-3}+1.437 \times 10^{-4} t-\right. \\
\left.\quad 3.071 \times 10^{-7} t^{2}+1.686 \times 10^{-10} t^{3}\right)\end{array}$ & $\begin{array}{l}\text { Moore et al. 2016; function } \\
\text { derived in this article }\end{array}$ \\
\hline Units of $i$ & $\begin{array}{l}\text { Energetic reserves provided } \\
\text { by one prey item }\end{array}$ & 5 calories & Riechert 1991 \\
\hline$c_{1}$ & $\begin{array}{l}\text { Fraction of } i \text { lost per } t \text { due } \\
\text { to resting metabolism }\end{array}$ & $5.21 \times 10^{-4}$ & $\begin{array}{l}\text { Greenstone and Bennett } \\
\text { 1980; Gnaiger } 1983\end{array}$ \\
\hline$c_{2}$ & $\begin{array}{l}\text { Fraction of } i \text { lost per } t \text { due } \\
\text { to metabolic costs of foraging }\end{array}$ & $4 \times c_{1}$ & Wilder 2011 \\
\hline$m$ & $\begin{array}{l}\text { Probability that predator encoun- } \\
\text { ter results in predation }\end{array}$ & .5 & na \\
\hline$f$ & $\begin{array}{l}\text { Probability that an attack is suc- } \\
\text { cessful (prey capture efficacy) }\end{array}$ & Varied, $\{.4, .8\}$ & na \\
\hline
\end{tabular}


by the number of time steps per interval (in this case, approximately 126). These data are deposited in the Dryad Digital Repository: https://dx.doi.org/10.5061/dryad.959g2 (Watts et al. 2017). We then estimated continuous probability functions for predator $(P(t))$ or prey item $(R(t))$ encounters by applying polynomial regressions (using the poly function in $\mathrm{R}$ v. 3.3.1) to the encounter probabilities estimated for each 3-h interval. For each function, we determined the degree of polynomial that best described the observed patterns of encounter probabilities by comparing models fit using polynomials of order 1-5 using the Akaike information criterion (see appendix, sec. 1). For patterns of both predator $(P(t))$ and prey $(R(t))$ encounters, third-order polynomials were best supported in the model selection procedure (fig. 2; appendix, sec. 1). For any times of day in which the best-fitting functions predicted negative encounter probabilities, we assumed that the encounter probability was 0 (table 1 ).

We used data on basal metabolic rate of the sister taxon to C. turbinata, Cyclosa conica, from Greenstone and Bennett (1980) to estimate the loss of energy reserves due to metabolism in each time step, $c_{1}$. We converted the rate reported in microliters of oxygen consumed per hour to an estimated number of calories burned per time step using equations from Gnaiger (1983). Assuming a unit of energy reserves in our model corresponds to the energetic content of typical, small flying insect prey (i.e., $\sim 1.25 \mathrm{cal}-$ ories; Riechert 1991), we then express basal metabolic rate, $c_{1}$, as a fraction of a 1.25-calorie energy unit. Thus, we estimate basal metabolic rate $c_{1}$ to be $5.21 \times 10^{-4}$ prey items per 85.8-s time step. These data are deposited in the Dryad Digital Repository: https://dx.doi.org/10.5061/dryad.959g2 (Watts et al. 2017). To obtain $c_{2}$, we estimated the value $s$ by which $c_{1}$ increases as a result of leaving the web hub to subdue a prey item $\left(c_{2}=s c_{1}\right)$. In spiders, activities such as foraging can increase metabolic rates two- to sixfold (Wilder 2011). Our analyses indicate that varying $s$ within this range does not affect qualitative model predictions, so we report results for the average $s$ value of 4 .

Our general model rests on the assumption that increases in energetic reserves cause increases in relative fitness, although the precise mechanisms of this relationship are likely system specific. In C. turbinata, diet manipulations conducted under natural conditions show that experimentally increasing prey capture leads to an increase in the number of eggs produced by females (Spiller 1984). Consequently, for our case study we assume that relative fitness is an increasing function of energetic reserves due to effects of energetic reserves on fecundity. Thus, the parameterization of the model for C. turbinata effectively maximizes relative fecundity. As described in Fitness Currency, we consider the full range of possible energetic states and therefore assume that the relationship between energetic reserves and

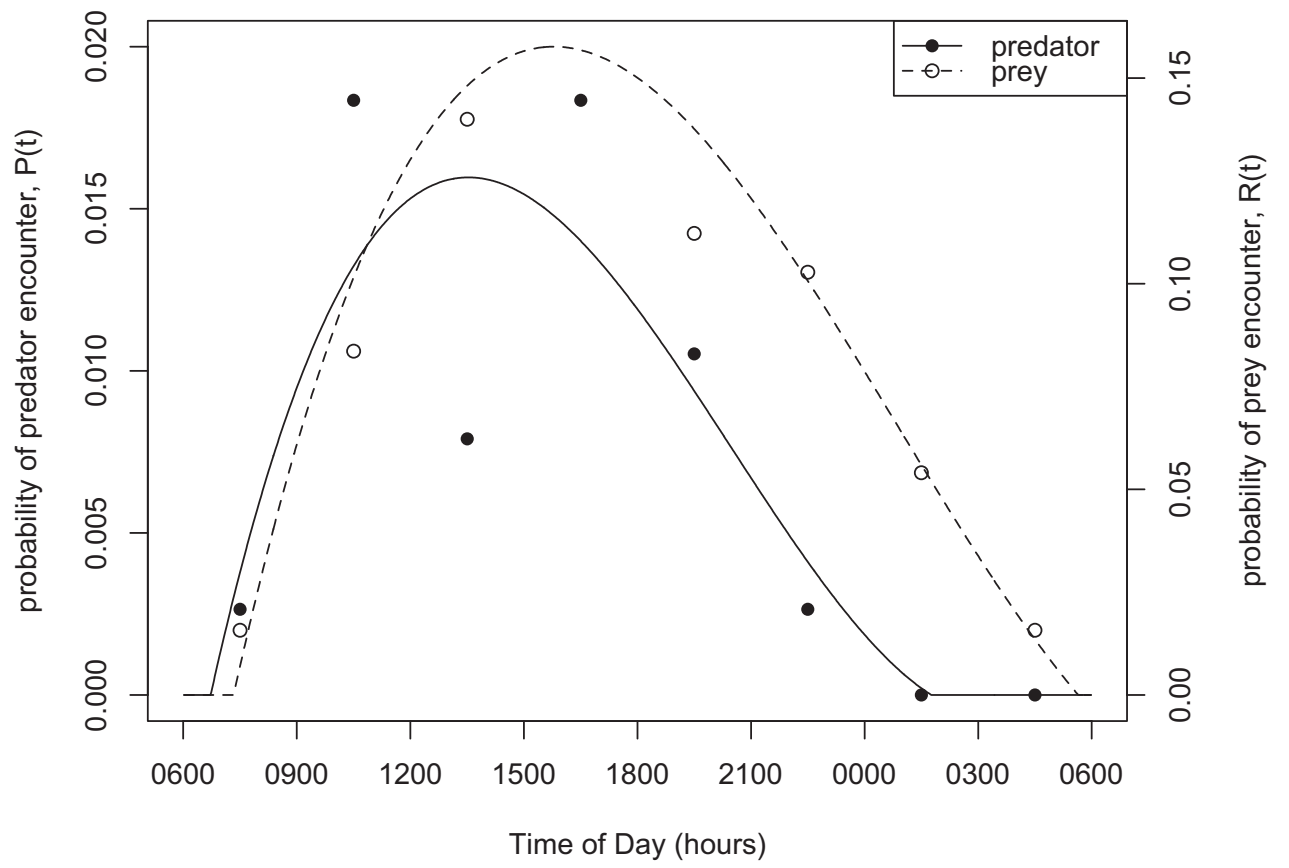

Figure 2: Probability of encountering a visual hymenopteran predator (solid line) or a prey item (dashed line) as a function of time of day. Probability functions are estimated using average probabilities of predator encounters (filled circles) and prey encounters (open circles) over 3-h periods derived from encounter rates at flight interception traps placed in Cyclosa turbinata habitat (Moore et al. 2016). 
fitness (i.e., relative fecundity) is sigmoidal. A sigmoidal fitness function accounts for changes in marginal fitness payoffs due to (i) possessing energetic reserves already sufficient to nearly maximize fecundity and (ii) possessing energetic reserves so low that marginal increases in resources that can be allocated to reproduction do not increase the number of viable offspring. For our case study described below, relaxing this assumption does not affect the qualitative model predictions (see appendix). We therefore show results only for a sigmoidal fitness function of the form $F(i)=$ $\left.1 /\left(1+e^{-0.125 \times(i-50)}\right)\right)$, such that the inflection point is located at $50 \%$ of maximum energetic reserves (i.e., $i=50$ ).

\section{Model Analysis}

Using the parameters described above, we solve the model to determine the combination of states (energetic reserves $i$ and times of day $t$ ) at which female C. turbinata should forage to maximize the expected energy reserves available for producing eggs while minimizing predation risk ( $\mathrm{R}$ code deposited in the Dryad Digital Repository: https:// dx.doi.org/10.5061/dryad.959g2 [Watts et al. 2017]). In this article, we consider the energy threshold below which individuals start foraging a measure of boldness because foraging in the presence of predators is risky. This implies that, of two individuals with the same energy state, the one that forages during the riskiest period of the day is bolder than the resting individual. We then explore the sensitivity of the model predictions to changes in parameter values and model assumptions to determine whether the predicted pattern of behavior represents a general solution to the foragingpredation risk trade-off in C. turbinata and to what extent the conclusions of our model may apply to a wide range of species.

First, we recognize that $R(t)$ and $P(t)$ likely represent somewhat upwardly biased encounter probabilities, if for no other reason than the larger surface area of flight interception traps relative to a C. turbinata web. Moreover, predation risk $\mu(t)$ depends on not only a predator encounter $P(t)$ but also the probability $m$ that an encounter ends in predation (see Predation Risk and Prey Availability). Similarly, the overall probability of capturing a prey item depends on both the probability of encountering prey $R(t)$ and the individual's prey capture efficacy $f$ (i.e., the probability that an attack is successful). We currently have no strong empirical data to inform our estimates of $m$ and $f$ under natural conditions. Consequently, we vary the magnitude of $\mu(t), R(t)$, and $f$ to simulate the effect of changes in overall predation risk, prey abundance, and prey capture efficacy on predicted patterns of foraging behavior. Because the large flight-interception traps likely overestimate rates of predator and prey encounters at the web, we consider $\mu(t)$ and $R(t)$ with magnitudes ranging from $10 \%$ to $90 \%$ of the estimates obtained from the traps except where noted. We also consider scenarios where $\mu(t)$ or $R(t)$ is uniform across the day to assess the relative contribution of patterns of prey and predator abundance to the predicted rhythm of behavior.

Second, we acknowledge that the predictions of the model may depend on the ratio of metabolic rate to energy content of prey. We vary the basal metabolic rate $c_{1}$ and, as a result, the metabolic rate during foraging $c_{2}$ to determine the robustness of our model to our estimate of metabolic rate in C. turbinata. By further varying basal metabolic rate outside the range likely for $C$. turbinata, we also assess whether the model predictions are likely to apply to sit-and-wait predators with higher metabolic rates than those of spiders.

Finally, we further vary predation risk, prey availability, and metabolic rate parameters beyond the range likely to occur in C. turbinata to assess the generality of the model for organisms that face different physiological or ecological constraints.

\section{Foraging Observations}

To determine whether foraging behavior under natural conditions is consistent with our qualitative model predictions, we used data from previous recordings of individuals in the field. Specifically, C. turbinata foraging behavior was recorded for 10 consecutive days at the field site described above (see Parameterizing the Model). We collected 13 female C. turbinata from within $8 \mathrm{~km}$ of the field site and placed them in open-sided wooden frames $(30 \mathrm{~cm} \times$ $10 \mathrm{~cm} \times 30 \mathrm{~cm}$ ) that were then mounted along a wooden support structure to facilitate the attachment of a video surveillance camera system capable of infrared recording in the dark (Q-See 16-channel HD security system). Four of the 13 individuals abandoned the wooden frames within the first few days of observation. Consequently, we obtained 10 days of continuous video observations of nine female C. turbinata. Using these videos, we recorded the time of day of each instance of a prey item hitting the web and whether the individual attacked the prey item. These data are deposited in the Dryad Digital Repository: https://dx.doi .org/10.5061/dryad.959g2 (Watts et al. 2017). We then used a binomial generalized additive model (GAM) with logit link (using the gam function in the mgcv package [Wood 2006] for R v. 3.3.1 [R Core Team 2016]) to analyze the relationship between the time of day that an insect hits the web (entered as a continuous predictor) and individuals' foraging decisions (attack/rest), using an additional smoothing term to account for individual identity and day random effects (Wood 2006). Based on our model predictions, foraging is optimal across virtually all energetic states between approximately 0100 and 0600 hours, but resting is optimal for at least some states from approximately 0600 to 0100 hours (see Results); therefore, if at least some individuals were in a high energetic 
state during our observations, we expect the likelihood of attacking to generally decrease with time of day (hours).

\section{Results \\ Model Analysis}

The SDP model determines for each combination of states the optimal behavioral decision, forage or rest. We then determine for each time of day the level of energetic reserves above which it becomes optimal to rest (hereafter, the "energy threshold"). This energy threshold represents the optimal behavioral policy (i.e., if energy reserves lie above the threshold, rest; otherwise, forage). Because the differences in fitness between foraging and resting for individuals near the energy threshold can become very small toward the end of the day and introduce rounding errors when determining the optimal behavior (resulting in an unrealistically ragged energy threshold), we assume that individuals forage by default unless the fitness benefits of resting exceed that of foraging by $2 \times 10^{-4}$ fitness units (or $0.02 \%$ of the maximum fitness). This small buffer removes rounding artifacts from the model predictions. To determine whether a foragingpredation risk trade-off can produce selection for daily rhythms of foraging behavior, we visualize the energy threshold over the day to determine whether the optimal behavioral policy varies over the daily cycle. Stated differently, temporal changes in the location of the energy threshold suggest that the decision that maximizes fitness depends on the time of day for at least some energetic states.

Our model results support the hypothesis that temporal variation in a foraging-predation risk trade-off can select for daily patterns of foraging behavior, as evidenced by a strong temporal pattern in the energy threshold. Our model predicts that female Cyclosa turbinata should generally start foraging at higher levels of energy reserves (i.e., should be more bold) during the early morning and late night than during the daytime when predator abundance peaks (fig. 3). Specifically, if hymenopteran predator abundance is low during the night, individuals should attack encountered prey during the early morning and late night irrespective of energetic reserves. In contrast, females' decision to attack encountered prey during the daytime depends on energetic reserves for most parameter combinations.

Changes in the average magnitude of predation risk, $\bar{\mu}(t)$, have a subtle effect on the model predictions. Under all levels of predation risk considered here $(10 \%-90 \%$ of the predation risk estimated from flight interception traps; Moore et al. 2016), females should stop foraging at lower levels of energy reserves during the midday period; however, the exact level of the energy threshold depends on the magnitude of predation risk (fig. $4 A$ ). In general, as the average magnitude of predation risk increases, the model predicts that females should stop foraging during the midday pe-

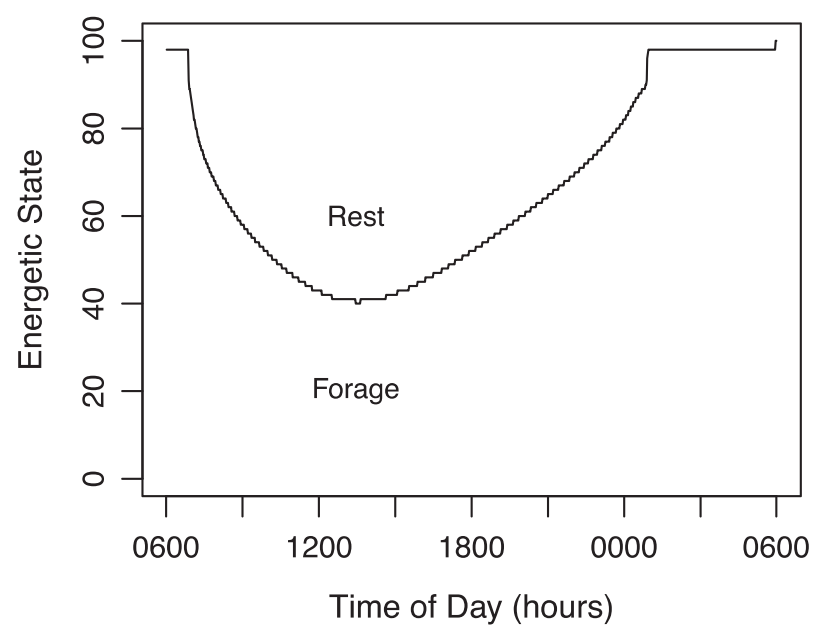

Figure 3: Predictions of the stochastic dynamic programming model of daily foraging behavior in the trashline orb weaver, Cyclosa turbinata. The boundary represents the level of energetic reserves above which the optimal decision for a female spider is to remain at rest at the web hub at the expense of capturing prey ensnared in the web (i.e., the energy threshold). Here, prey capture efficacy $f=0.5$; all other parameter values are presented in table 1.

riod even if energetic reserves are relatively low (i.e., females should be shier). This follows intuitively from the additional, time-dependent predation risk associated with leaving the decoy to forage. Increasing the magnitude of this risk decreases the range of conditions over which the risk of predation is outweighed by potential energy gains.

Similar to the effect of increasing predation risk, the probability of encountering prey $R(t)$ affects the quantitative, but not qualitative, model predictions. For all levels of prey availability considered here (10\%-90\% of the prey availability estimated from flight interception traps; Moore et al. 2016), females' energy thresholds should be lower during the risky midday period than during the early morning or night. As the average probability of encountering prey in the web, $\bar{R}(t)$, increases, the model generally predicts that the energy threshold during midday decreases (i.e., females should be shier; fig. $4 B$ ). As prey encounters increase in frequency, there are more opportunities to gain energy through foraging, and thus gaining energy during the high-risk midday period becomes less critical for maximizing fitness over the daily cycle.

The effect of prey capture efficacy $f$ on the quantitative model predictions depends on prey abundance and predation risk; thus, to better understand the effect of $f$, we compare the energy threshold between a female with $f=0.8$ and a female with $f=0.4$ during the time when predation risk is greatest ( $t=316$, or 1331 hours) over a range of average prey encounter probabilities for two different values of predation risk (fig. 5). We vary prey availability by scal- 

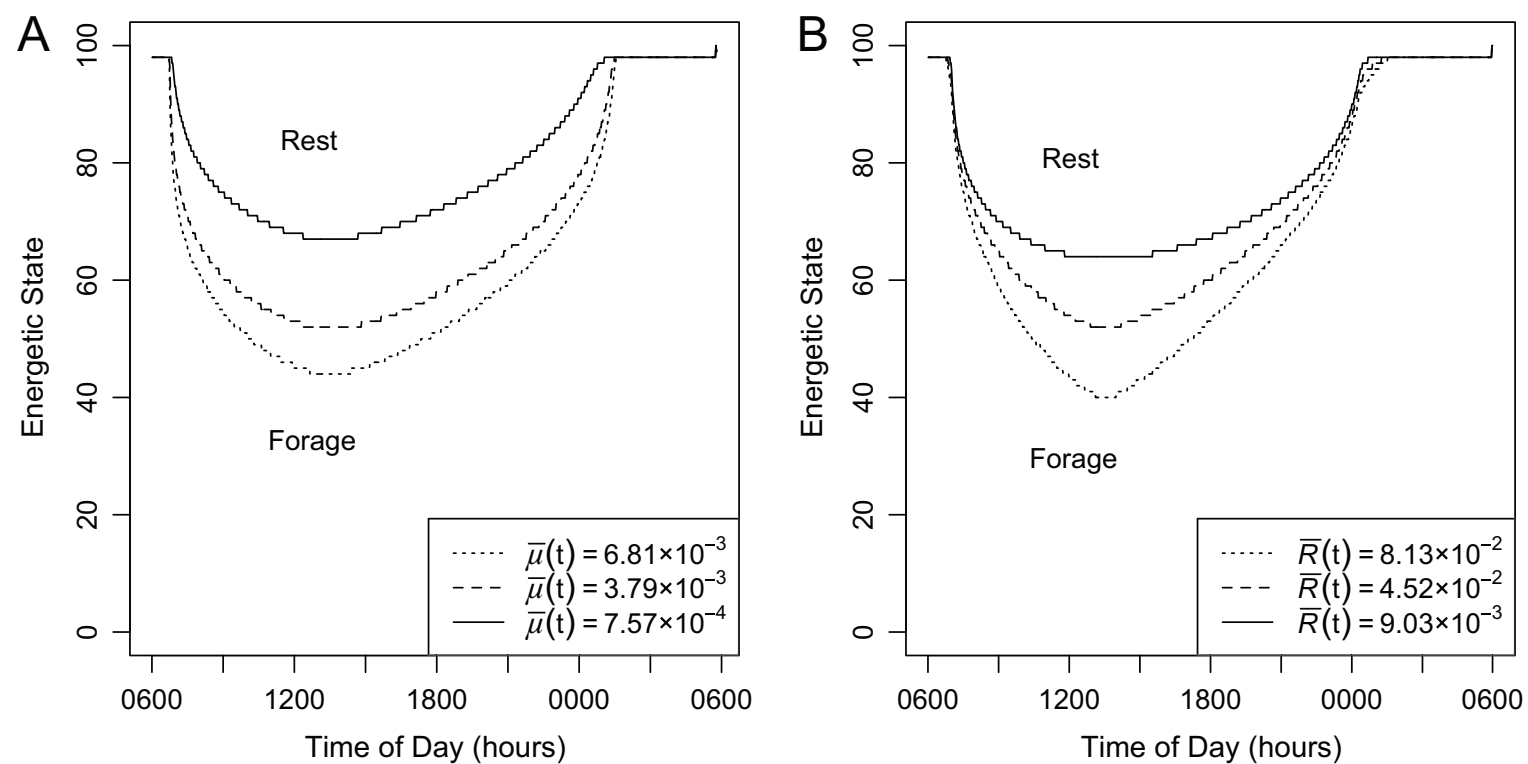

Figure 4: Effect of predation risk $(A), \bar{\mu}(t)$, and the probability of encountering prey $(B), \bar{R}(t)$, on the pattern of boldness predicted by the model. Lines represent the energy threshold (see fig. 3) for each parameter value. The relative daily patterns of $\mu(t)$ and $R(t)$ are the same in each case; only the magnitude (and thus the mean, $\bar{\mu}(t)$ or $\bar{R}(t)$ ) of the function differs. In $A, \bar{R}(t)=4.52 \times 10^{-2}$, and in $B, \bar{\mu}(t)=3.79 \times$ $10^{-3}$. In all cases, prey capture efficacy $f=0.5$, and all other parameter values are as in table 1.

ing the prey encounter probability function $R(t)$ by a scaling factor $s \in(0,1]$, which implies that a female will encounter prey in the next time step with probability $R(t) \times s$. The two values of average predation risk, $\bar{\mu}(t)=1.89 \times 10^{-3}$ and $\bar{\mu}(t)=9.46 \times 10^{-3}$, represent $25 \%$ and $125 \%$, respectively, of the average predation risk estimated from flight interception traps (Moore et al. 2016). We find that if prey encounters are relatively infrequent, the model predicts

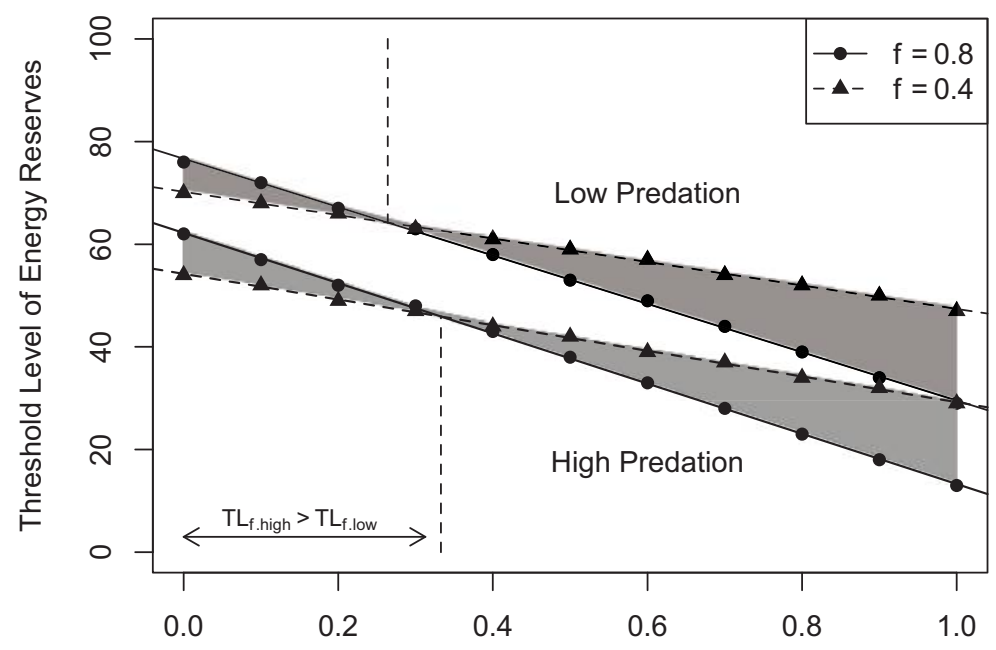

Relative Prey Availability

Figure 5: Threshold level of energetic reserves for an effective forager ( $f=0.8$; solid lines with circles) and a less effective forager ( $f=0.4$; dashed lines with triangles) at the time of day when risk reaches a maximum $(t=316$, or 1330 hours $)$ over a range of average prey availability $\left(X\right.$-axis) for both low and high average predation risk scenarios $\left(\bar{\mu}(t)=1.89 \times 10^{-3}\right.$ and $\bar{\mu}(t)=9.46 \times 10^{-3}$; upper and lower pairs of lines, respectively). The shaded region represents the magnitude of the effect of $f$ for a given value of prey abundance and predation risk. The dashed vertical lines delineate the level of prey availability at which the threshold level of energy reserves $(\mathrm{TL})$ of a less effective forager $\left(\mathrm{TL}_{\text {flow }}\right)$ exceeds that of a more effective forager $\left(\mathrm{TL}_{f . \text { high }}\right.$ ), which depends on predation risk. 
that females' energy thresholds during the midday period should increase as prey capture efficacy $f$ increases (i.e., more effective females should be bolder; fig. 5). In contrast, if prey encounters are relatively frequent, females' energy thresholds should decrease as prey capture efficacy $f$ increases (i.e., more effective females should be shier; fig. 5). The range of prey abundances over which a more effective forager should be bolder increases with increasing predation risk (fig. 5).

The effect of prey capture efficacy $f$ arises because $f$ affects both the probability that any single foraging attempt will result in energetic gain and the overall rate of prey capture over the daily cycle. We have already shown that the rate of prey capture affects the optimal level of boldness during the high-risk period by varying $\bar{R}(t)$ (fig. $4 B$ ). However, we find a stronger decrease in boldness with increasing prey abundance for females with higher prey capture efficacy (fig. 5). The effect of $f$ on the response to prey abundance implies that the energy threshold of a more effective forager is at most equal to that of a less effective forager; however, $f$ also determines the probability that a foraging attempt will offset the risk of predation through prey capture. Accordingly, we find that the effect of predation risk (i.e., to decrease boldness; fig. $4 A$ ) is indeed weaker for more effective foragers (fig. 5). In our scenario, increasing predation risk results in a decrease in the energy threshold (change in the intercept of the linear model fit; fig. 5) of about 14 units for more effective foragers and about 16 units for less effective foragers. Because more effective foragers are less sensitive to predation risk, the difference in boldness between more and less effective foragers is generally less than predicted based on differential responses to prey abundance alone. Combined with differential responses to prey abundance, this effect leads to the prediction that more effective foragers should be bolder than less effective foragers when prey abundance is low. This also explains why the range of prey abundances over which a more effective forager should be bolder increases with predation risk (fig. 5). Because less effective foragers show a greater decrease in boldness with increasing predation risk, the level of prey availability necessary to decrease the boldness of effective foragers to that of less effective foragers increases as predation risk increases.

For C. turbinata, the qualitative model predictions are robust to variation in the estimate of metabolic rate $c_{1}$. Intuitively, as metabolic rate increases, the model predicts that females' energy thresholds should increase, reflecting the need to garner more energy before surpassing maintenance costs and developing reserves allocable to egg production. However, the magnitude of this effect is small and quickly becomes negligible for estimates of $c_{1}$ less than that obtained from the literature for C. turbinata (appendix, sec. 5). Based on the literature (Greenstone and Bennett 1980), there is no biologically reasonable estimate of metabolic rate for $C$. turbinata for which the model does not predict that individuals should have higher energy thresholds (i.e., should be more bold) during the early morning and late night.

Daily patterns of predation risk, not prey availability, drive the pattern of behavior predicted by the model (appendix, sec. 6). If both predation risk $\mu(t)$ and the probability of encountering prey $R(t)$ remain constant over the daily cycle, the predicted energy threshold remains constant. Incorporating the daily pattern of prey availability alone does not influence this qualitative prediction. Only when incorporating the temporal pattern of predation risk due to foraging does the model predict that individuals should forage in the early morning and over the night independent of energetic reserves yet forage during the daytime only if energetic reserves fall below a critical value. This effect persists even when $R(t)$ does not change over the daily cycle. This is because our model applies to sitand-wait predators that do not actively search for prey. Because individuals can only choose to forage or rest once prey have been encountered, they must balance current predation risk against the long-term risk of not acquiring enough prey, as the availability of a prey item at the moment of the decision is certain (we ignore prey detection mistakes for simplicity). However, for cases in which predators must search for their prey (described in appendix, sec. 2), both daily patterns of predation risk and prey availability contribute to the daily pattern predicted by the model (appendix, sec. 6). The sensitivity of searching predators to patterns of prey availability arises due to the decreased profitability of foraging when prey are scarce and provides one possible qualitative difference between model predictions for sit-and-wait predators and searching predators.

\section{General Considerations}

We provide a preliminary assessment of the generality of our conclusions for a wider range of organisms by further varying parameters outside the range of values likely to occur for C. turbinata. Specifically, we assess the influence of lower and higher values ( $<10 \%$ and $>90 \%$ of our estimates) of predation risk, prey availability, and metabolic rate, as these parameters are likely to vary most widely among study systems. In general, we find that our conclusions extend well beyond the range of conditions experienced by C. turbinata (see appendix).

\section{Foraging Observations}

The model predictions for C. turbinata generally reflect the pattern of foraging behavior described in a previous study conducted under controlled laboratory conditions (Watts et al. 2014). By simulating prey vibrations in the 
web at different points in the daily cycle, Watts et al. (2014) found that female C. turbinata more frequently attacked prey during the early morning and night than during the middle of the day, but females did not universally ignore prey during the day. Based on our model predictions, females' tendency to ignore prey during the day depends on energy reserves, so it is possible that the energy reserves of some females dropped below the energy threshold below which it is optimal to attack prey. However, because females were fed two termite workers each day prior to the experiment, the energy reserves of most females were likely above this threshold, resulting in resting during the day.

In the field, we observed patterns of prey encounters at the web similar to those described for malaise trap data from Moore et al. (2016; fig. 6A), though we failed to detect any incidences of predation. Nonetheless, we found a significant effect of time of day on the likelihood of a spider attacking prey that hit the web (binomial GAM: $\chi^{2}=$ 7.15; estimated $\mathrm{df}=1$; reference $\mathrm{df}=1 ; P=.008$; fig. $6 B$ ). As predicted, females always attacked prey during the early morning and more frequently ignored prey during the day. Specifically, the only instances in which females rested instead of attacking occurred between 1000 and 2200 hours. Though we failed to recover statistical support for an increase in boldness toward the very end of the day, this may be due to the low frequency of prey encounters between 2200 and 0000 hours. More generally, our prediction that individuals should be less likely to attack during the day rests on the assumption that some proportion of individuals achieve a high enough energetic state to exceed the threshold during the high-risk period. Consequently, a rigorous test of our model would require manipulating or quantifying individual energetic state. For instance, we expect that those spiders that ignore prey during the middle of the day should be in a higher energetic state than those that attack prey during this time.

\section{Discussion}

Despite the simplicity of the predation risk allocation hypothesis (PRAH; Lima and Bednekoff 1999) and the probable ubiquity of temporal variation in foraging-predation risk trade-offs, there remain few studies in which the plausibility of this mechanism for generating daily patterns of behavior has been assessed by testing the predictions of mechanistic models under natural conditions (but see Rosland and Giske 1994; Metcalfe et al. 1999; Tarling et al. 2000; Jensen et al. 2006), and to our knowledge, no such studies have been conducted for terrestrial organisms. By parameterizing our model using published data on ecological and physiological/energetic parameters, we find that
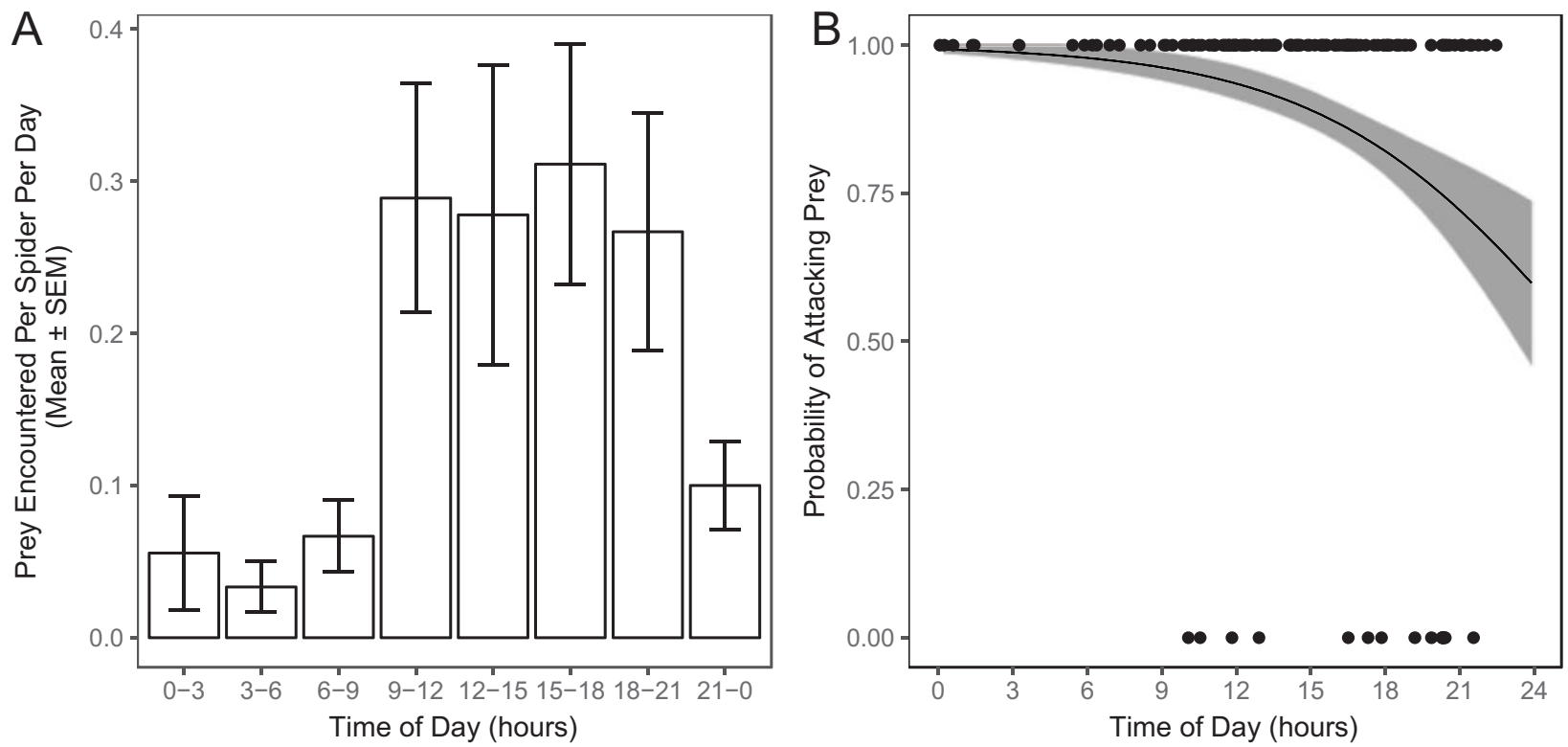

Figure 6: $A$, Daily pattern of the mean number of prey items intercepted by webs for nine spiders recorded continuously for 10 days under natural conditions. $B$, Probability that the spider attacked prey items intercepted by the web depended on the time of day (binomial generalized additive model: $\chi^{2}=7.15, P=.008$ ). Points represent the observed behavior (attack or rest) for each occurrence of a prey item hitting a spider's web. The line and shaded region represent the predicted probability of attacking ( $\pm 95 \%$ confidence interval) as a function of time of day. 
the PRAH provides a plausible explanation for daily rhythms of foraging behavior in an orb-weaving spider observed in the laboratory and under natural conditions and that such a trade-off can contribute to selection for daily patterns of foraging across a wide range of ecological scenarios, including those in which predators must actively search for their prey. While similar qualitative predictions regarding relative changes in behavior can be obtained by comparing ratios of risk to reward across the daily cycle (Lima and Bednekoff 1999; Metcalfe et al. 1999), modeling the optimal behavioral decision as a function of energetic state reveals additional implications of the risk-allocation problem.

First, our model predicts that the optimal solution to the daily risk-allocation problem is generally state dependent, suggesting that the same ecological mechanism that produces selection for rhythmicity also favors the ability to suppress rhythmicity when individuals are in a low energetic state. Consequently, findings that seem to contradict the PRAH (e.g., a lack of foraging rhythm in individuals that experience temporal variation in risk) may in fact be consistent with the core predictions of this hypothesis, and thus demonstrating the effect of individual state on the daily rhythm should generally be critical for rigorous assessments of the role of risk allocation in shaping behavioral strategies.

Second, our model predicts that environmental parameters (i.e., predation risk and prey encounter rate) and internal parameters (i.e., prey capture efficacy) interact to determine the optimal behavior. Specifically, more effective foragers should be relatively more sensitive to changes in prey encounter rates, whereas less effective foragers should be relatively more sensitive to predation risk, and these differences can lead to changes in the qualitative effect of differences in foraging efficacy. These complex interactions highlight the importance of thoroughly exploring predictions of an adaptive hypothesis prior to assessing the supporting empirical evidence. For example, researchers seeking to test the role of risk allocation in a given taxonomic group might do so by comparing behavioral rhythms between habitats that differ in mean predation risk or between individuals that differ in their efficacy as foragers. Our model suggests that the quantitative or even qualitative differences in behavior expected in such studies likely depend on the values of the remaining environmental or internal parameters. Thus, the interpretability of such studies may rely on obtaining estimates of additional parameters that might otherwise be dismissed as irrelevant provided they are controlled through experimental design.

For our case study, the model predicts that female Cyclosa turbinata should be bolder (i.e., forage over a greater range of energetic states) during the morning and evening when there is little risk of encountering hymenopteran predators while away from the web decoy. This predicted pattern of behavior reflects those observed under laboratory conditions (Watts et al. 2014), and we also found that individuals observed in their natural habitat ignore prey more frequently during the high-risk daytime period than during the early morning. Although these patterns of behavior are consistent with our model of risk allocation, as we note above, our model also suggests that a strong test of the predictions would require demonstrating the effect of energetic state on foraging behavior. As we recorded individuals under natural conditions prior to developing our model, we did not attempt to assess or manipulate energetic state in our observations.

Our model also provides generalizable insight into the dynamics of consistent variation in behavior among individuals facing similar conditions. While among-individual behavioral variation might be dismissed as noise around a behavioral optimum, such variation is often highly consistent through time (Sih et al. 2004, 2012). Consequently, consistent differences in behavior (e.g., boldness) have garnered considerable attention from behavioral ecologists seeking to understand the processes that generate and maintain this variation (Sih et al. 2004, 2012). As described above, our model shows that among-individual variation in foraging behavior can arise from state-dependent solutions to the trade-off between foraging and predation risk (e.g., between fed and hungry females). However, the dynamics we uncover reflect aspects of the asset protection principle (Clark 1994; Wolf et al. 2007), in which individuals who garner resources become shier (more risk-averse), while individuals that fail to garner resources become bolder (more risk-prone). Such negative feedback between state and boldness may act to reduce variation in state (e.g., energy reserves) among individuals under some conditions (McElreath et al. 2007; Luttbeg and Sih 2010). Nevertheless, differences in state variables that influence behavior may be maintained to some degree by positive feedback mechanisms such as positive state-dependent safety (Luttbeg and Sih 2010). In C. turbinata, the web decoy is formed in part from remains of prey items and may therefore represent a positive feedback between current energetic state and future safety. Moreover, our model demonstrates that foraging efficacy, which may be influenced by genetic or environmental factors that are less dynamic than energetic state, may also produce among-individual differences in daily patterns of behavior. Considering such dynamics with respect to daily rhythms may reveal mechanisms that generate and maintain among-individual variation in boldness over the daily cycle despite strong rhythms of individual behavior (Watts et al. 2014, 2015). Additionally, because individuals that differ in prey capture efficacy should differ in the strength of their responses to environmental conditions (see above), this effect may provide an adaptive mechanistic explanation for correlations observed between among-individual variation in behavior and among-individual variation in behavioral plasticity across a wide diversity of taxa (Dingemanse et al. 2009). 
We also briefly considered a scenario in which foragers must search for prey and demonstrated one intuitive difference between searching foragers and sit-and-wait foragers. Searching foragers are sensitive to the temporal pattern of both predation risk and prey encounters, whereas sit-and-wait foragers are sensitive only to the temporal pattern of predation risk. However, foragers that must search for their prey may differ from sit-and-wait foragers with respect to a variety of model parameters (e.g., handling time, metabolic costs of foraging, or predation risk), and a thorough exploration of these scenarios exceeds the scope of this study. Additionally, because our model does not make assumptions about physiological processes underlying daily changes in trait expression (e.g., endogenous rhythms), our general approach should apply to organisms that experience rhythmic variation in risk at a variety of temporal scales. Consequently, further research on how the predictions of the PRAH differ among foraging strategies, as well as the extent to which the PRAH can explain other biological rhythms, such as tidal, lunar, or annual rhythms, should greatly enhance our understanding of the ultimate causes of rhythmicity in living systems.

\section{Acknowledgments}

We thank East Tennessee State University Department of Biological Sciences staff for logistical support. We thank Darrell Moore for invaluable intellectual input in the early stages of this work. We also thank the numerous faculty and graduate students of the Ecology, Evolution, and Behavior group in the University of Nebraska-Lincoln School of Biological Sciences who provided feedback on this article. We are particularly indebted to two anonymous reviewers whose comments greatly improved the clarity of our article. This work was funded in part by National Science Foundation Division of Integrative Organismal Systems award 1257133.

\section{Literature Cited}

Aschoff, J. 1981a. A survey of biological rhythms. Pages 3-10 in J. Aschoff, ed. Handbook of behavioral neurobiology. Vol. 4. Biological rhythms. Plenum, New York.

1981b. Free-running and entrained circadian rhythms. Pages 81-93 in J. Aschoff, ed. Handbook of behavioral neurobiology. Vol. 4. Biological rhythms. Plenum, New York.

Beaver, L. M., B. L. Rush, B. O. Gvakharia, and J. M. Giebultowicz. 2003. Noncircadian regulation and function of clock genes period and timeless in oogenesis of Drosophila melanogaster. Journal of Biological Rhythms 18:463-472.

Bertram, S. M., S. X. Orozco, and R. Bellani. 2004. Temporal shifts in conspicuousness: mate attraction displays of the Texas field cricket, Gryllus texensis. Ethology 110:963-975.

Blackledge, T. A., J. A. Coddington, and R. G. Gillespie. 2003. Are three-dimensional spider webs defensive adaptations? Ecology Letters 6:13-18.
Chou, I. C., P. H. Wang, P. S. Shen, and I. M. Tso. 2005. A test of preyattracting and predator defence functions of prey carcass decorations built by Cyclosa spiders. Animal Behaviour 69:1055-1061.

Clark, C. W. 1994. Antipredator behavior and the asset-protection principle. Behavioral Ecology 5:159-170.

Clark, C. W., and M. Mangel. 2000. Dynamic state variable models in ecology: methods and applications. Oxford University Press, Oxford.

DeCoursey, P. J. 2004. The behavioral ecology and evolution of biological timing systems. Pages 27-66 in J. C. Dunlap, J. J. Loros, and P. J. DeCoursey, eds. Chronobiology: biological timekeeping. Sinauer, Sunderland, MA.

Dingemanse, N. J., A. J. N. Kazem, D. Reale, and J. Wright. 2009. Behavioural reaction norms: animal personality meets individual plasticity. Trends in Ecology and Evolution 25:81-89.

Emens, J. S., K. Yuhas, J. Rough, N. Kochar, D. Peters, and A. J. Lewy. 2009. Phase angle of entrainment in morning- and evening-types under naturalistic conditions. Chronobiology International 26:474-493.

Enright, J. T. 1970. Ecological aspects of endogenous rhythmicity. Annual Review of Ecology and Systematics 1:221-238.

Fleury, F., R. Allemand, F. Vavre, P. Fouillet, and M. Bouletreau. 2000. Adaptive significance of a circadian clock: temporal segregation of activities reduces intrinsic competitive inferiority in Drosophila parasitoids. Proceedings of the Roval Society B 267:1005-1010.

Gnaiger, E. 1983. Calculation of energetic and biochemical equivalents of respiratory oxygen consumption. Pages 337-345 in E. Gniager and H. Forstner, eds. Polarographic oxygen sensors. Springer, Berlin.

Gonzaga, M. F., and J. Vasconcellos-Neto. 2005. Testing the functions of detritus stabilimenta in webs of Cyclosa fililineata and Cyclosa morretes (Araneae: Araneidae): do they attract prey or reduce the risk of predation? Ethology 111:479-491.

Greenstone, M. H., and A. F. Bennett. 1980. Foraging strategy and metabolic rate in spiders. Ecology 61:1255-1259.

Jensen, O. P., T. R. Hrabik, S. J. D. Martell, C. J. Walters, and J. F. Kitchell. 2006. Diel vertical migration in the Lake Superior pelagic community. II. Modeling trade-offs at an intermediate trophic level. Canadian Journal of Fisheries and Aquatic Sciences 63:2296-2307.

Johnson, C. H. 2005. Testing the adaptive value of circadian systems. Methods in Enzymology 393:818-837.

Kotler, B. P., J. Brown, S. Mukherjee, O. Berger-Tal, and A. Bouskila. 2010. Moonlight avoidance in gerbils reveals a sophisticated interplay among time allocation, vigilance and state-dependent foraging. Proceedings of the Roval Societv B 277:1469-1474.

Lima, S. L., and P. A. Bednekoff. 1999. Temporal variation in danger drives antipredator behavior: the predation risk allocation hypothesis. American Naturalist 153:649-659.

Luttbeg, B., and A. Sih. 2010. Risk, resources and state-dependent adaptive behavioural syndromes. Philosophical Transactions of the Roval Societv B 365:3977-3990.

Mangel, M. 1990. Dynamic information in uncertain and changing worlds. Journal of Theoretical Biology 146:317-332.

McElreath, R., B. Luttbeg, S. P. Fogarty, T. Brodin, and A. Sih. 2007. Evolution of animal personalities. Nature 450:E5.

McNamara, J. M., and A. I. Houston. 1985. Optimal foraging and learning. Journal of Theoretical Biology 117:231-249.

Metcalfe, N. B., N. H. C. Fraser, and M. D. Burns. 1999. Food availability and the nocturnal vs. diurnal foraging trade-off in juvenile salmon. Journal of Animal Ecology 68:371-381.

Moore, D. 2001. Honey bee circadian clocks: behavioral control from individual workers to whole-colony rhythms. Iournal of Insect Physiology 47:843-857. 
Moore, D., J. C. Watts, A. Herrig, and T. C. Jones. 2016. Exceptionally short-period circadian clock in Cyclosa turbinata: regulation of locomotor and web-building behavior in an orb-weaving spider. Journal of Arachnology 44:388-396.

Ouyang, Y., C. R. Andersson, T. Kondo, S. S. Golden, and C. H. Johnson. 1998. Resonating circadian clocks enhance fitness in cyanobacteria. Proceedings of the National Academv of Sciences of the USA 95: 8660-8664

Pittendrigh, C. S., and S. Daan. 1976. A functional analysis of circadian pacemakers in nocturnal rodents. IV. Entrainment: pacemaker as clock. Journal of Comparative Physiology 106:291-331.

R Core Team. 2016. R: a language and environment for statistical computing. R Foundation for Statistical Computing, Vienna.

Riechert, S. E. 1991. Prey abundance vs. diet breadth in a spider test system. Evolutionary Ecology 5:327-338.

Rietveld, W. J., D. S. Minors, and J. M. Waterhouse. 1993. Circadian rhythms and masking: an overview. Chronobiology International 10:306-312.

Rosland, R., and J. Giske. 1994. A dynamic optimization model of the diel vertical distribution of a pelagic planktivorous fish. Progress in Oceanography 34:1-43.

Sih, A., A. Bell, and J. C. Johnson. 2004. Behavioral syndromes: an ecological and evolutionary overview. Trends in Ecology and Evolution 19:372-378.

Sih, A., J. Cote, M. Evans, S. Fogarty, and J. Pruitt. 2012. Ecological implications of behavioural syndromes. Ecology Letters 15:278-289.

Sönnichsen, L., M. Bokje, J. Marchal, H. Hofer, B. Jedrzejewska, S. Kramer-Schadt, and S. Ortmann. 2013. Behavioural responses of European roe deer to temporal variation in predation risk. Ethology 119:233-243.

Spiller, D. A. 1984. Competition between two spider species: experimental field study. Ecology 65:909-919.
Tarling, G., M. Burrows, J. Matthews, R. Saborowski, F. Buchholz, A. Bedo, and P. Mayzaud. 2000. An optimisation model of the diel vertical migration of northern krill (Meganyctiphanes norvegica) in the Clyde Sea and the Kattegat. Canadian Journal of Fisheries and Aquatic Sciences 57:38-50.

Tseng, L., and I. Tso. 2009. A risky defence by a spider using conspicuous decoys resembling itself in appearance. Animal Behaviour 78:425-431.

Vaze, K. M., and V. K. Sharma. 2013. On the adaptive significance of circadian clocks for their owners. Chronobiology International 30:413-433.

Watts, J. C., A. Herrig, W. D. Allen, and T. C. Jones. 2014. Diel patterns of foraging aggression and antipredator behaviour in the trashline orb-weaving spider, Cyclosa turbinata. Animal Behaviour 94:79-86.

Watts, J. C., T. C. Jones, A. Herrig, M. Miller, and B. Tenhumberg. 2017. Data from: Temporal variation in predation risk may explain daily rhythms of foraging behavior in an orb-weaving spider. American Naturalist, Dryad Digital Repository, https://dx.doi.org/10.5061/dryad $.959 \mathrm{~g} 2$.

Watts, J. C., C. R. Ross, and T. C. Jones. 2015. Diel and life-history characteristics of personality: consistency versus flexibility in relation to ecological change. Animal Behaviour 101:43-49.

Wilder, S. M. 2011. Spider nutrition: an integrative perspective. Advances in Insect Physiology 40:88-125.

Wolf, M., G. S. Van Doorn, O. Leimar, and F. J. Weissing. 2007. Lifehistory trade-offs favour the evolution of animal personalities. Nature 447:581-584.

Wood, S. N. 2006. Generalized additive models: an introduction with R. CRC, Boca Raton, FL.

Associate Editor: Jürgen Groeneveld Editor: Yannis Michalakis

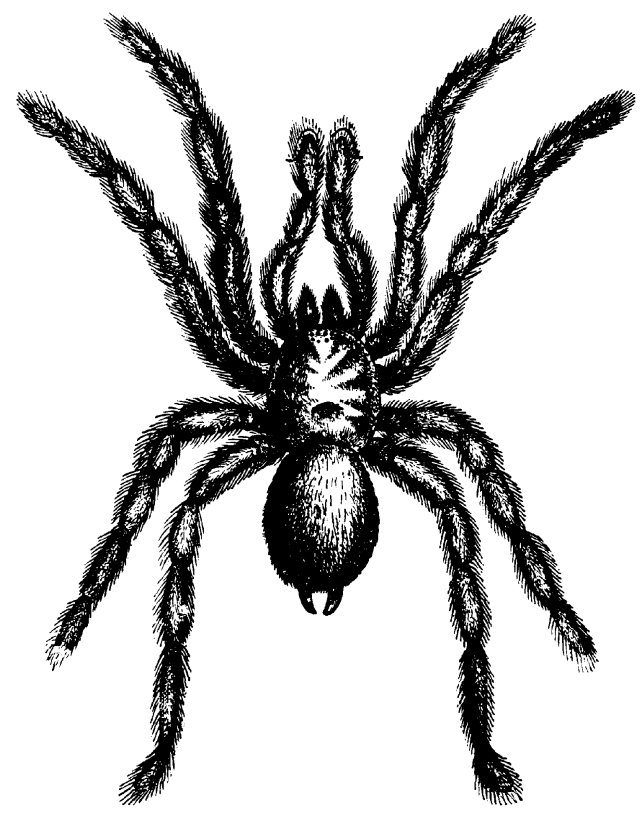

"The Pompilus, however, is a good-natured insect, showing no signs of pugnacity, except when she has a fine fat Tarantula in hand, and then she only threatens violence by spreading out her red wings, and running a little way towards the intruder." From "The Tarantula Killers of Texas" by G. Lincecum (The American Naturalist, 1867, 1:137-141). 